Marquette University

e-Publications@Marquette

$11-1-2016$

\title{
Conformational States of Cytochrome P450 Oxidoreductase Evaluated by Förster Resonance Energy Transfer Using Ultrafast Transient Absorption Spectroscopy
}

\author{
Elizaveta A. Kovrigina \\ Marquette University \\ Brian Pattengale \\ Marquette University \\ Chuanwu Xia \\ Medical College of Wisconsin \\ Azamat R. Galiakhmetov \\ Marquette University, azamat.galiakhmetov@marquette.edu \\ Jier Huang \\ Marquette University, jier.huang@marquette.edu
}

See next page for additional authors

Follow this and additional works at: https://epublications.marquette.edu/chem_fac

Part of the Chemistry Commons

\section{Recommended Citation}

Kovrigina, Elizaveta A.; Pattengale, Brian; Xia, Chuanwu; Galiakhmetov, Azamat R.; Huang, Jier; Kim, Jung $\mathrm{Ja}$; and Kovrigin, Evgueni, "Conformational States of Cytochrome P450 Oxidoreductase Evaluated by Förster Resonance Energy Transfer Using Ultrafast Transient Absorption Spectroscopy" (2016). Chemistry Faculty Research and Publications. 533.

https://epublications.marquette.edu/chem_fac/533 


\section{Authors}

Elizaveta A. Kovrigina, Brian Pattengale, Chuanwu Xia, Azamat R. Galiakhmetov, Jier Huang, Jung Ja Kim, and Evgueni Kovrigin

This article is available at e-Publications@Marquette: https://epublications.marquette.edu/chem_fac/533 


\title{
Conformational States of Cytochrome P450 Oxidoreductase Evaluated by Förster Resonance Energy Transfer Using Ultrafast Transient Absorption Spectroscopy
}

\author{
Elizaveta A. Kovrigina \\ Biochemistry Department, Medical College of Wisconsin, \\ Milwaukee, WI \\ Chemistry Department, Marquette University, \\ Milwaukee, WI \\ Brian Pattengale \\ Chemistry Department, Marquette University, \\ Milwaukee, WI \\ Chuanwu Xia \\ Biochemistry Department, Medical College of Wisconsin, \\ Milwaukee, WI \\ Azamat R. Galiakhmetov \\ Chemistry Department, Marquette University, \\ Milwaukee, WI
}

Biochemistry, Vol 55, No. 43 (November 1, 2016): pg. 5973-5976. DOI. This article is @ American Chemical Society and permission has been granted for this version to appear in e-Publications@Marquette. American Chemical Society does not grant permission for this article to be further copied/distributed or hosted elsewhere without the express permission from American Chemical Society. 
NOT THE PUBLISHED VERSION; this is the author's final, peer-reviewed manuscript. The published version may be accessed by following the link in the citation at the bottom of the page.

\author{
Jier Huang \\ Chemistry Department, Marquette University, \\ Milwaukee, WI \\ Jung-Ja P. Kim \\ Biochemistry Department, Medical College of Wisconsin, \\ Milwaukee, WI \\ Evgenii L. Kovrigin \\ Chemistry Department, Marquette University, \\ Milwaukee, WI
}

\title{
Abstract
}
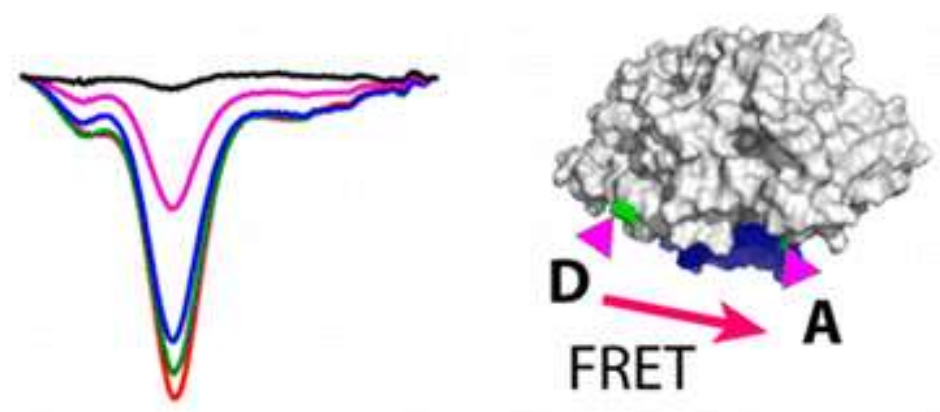

Transient Absorption FRET

NADPH-cytochrome P450 oxidoreductase (CYPOR) was shown to undergo large conformational rearrangements in its functional cycle. Using a new Förster resonance energy transfer (FRET) approach based on femtosecond transient absorption spectroscopy (TA), we determined the donor-acceptor distance distribution in the reduced and oxidized states of CYPOR. The unmatched time resolution of TA allowed the quantitative assessment of the donor-acceptor FRET, indicating that CYPOR assumes a closed conformation in both reduced and oxidized states in the absence of the redox partner. The described ultrafast TA measurements of FRET with readily available redinfrared fluorescent labels open new opportunities for structural studies in chromophore-rich proteins and their complexes.

NADPH-cytochrome P450 oxidoreductase (CYPOR) functions to transfer electrons from NADPH to various monooxygenases that are responsible for a wide range of cellular functions. X-ray diffraction 
studies of the cytosolic domain of CYPOR identified two conformations that are required for electron transfer (ET) from NADPH to electron acceptors such as cytochromes P450. ${ }^{1}$ One is the "closed" conformation allowing ET from NADPH to the enzyme-bound flavin cofactors; another is the "open" state interacting with its ET partner proteins, e.g., cytochromes P450 and heme oxygenase. 2,3 The two functions are mutually exclusive; CYPOR must cycle through the openclosed transitions to allow a continuous flow of electrons from NADPH to cytochrome P450 and other electron acceptors. This reaction cycle has been qualitatively monitored using Förster resonance energy transfer (FRET) between donors and acceptors nonspecifically attached to the endogenous surface cysteines in CYPOR, ${ }^{4,5}$ yet quantitative analysis has been lacking.

FRET measurement is a well-established approach for determining approximate distances between donor and acceptor sites in biomacromolecules and supramolecular assemblies. ${ }^{6-10}$ The most crucial requirement for quantitative FRET analysis is that the excitation and emission bands of the donor-acceptor pair occur in the isolated spectral region from the protein absorption bands. Otherwise, the unwanted resonance energy transfer to and from the intrinsic protein chromophores and fluorophores destroys the information content of the FRET experiment. This is particularly important in proteins involving ET reactions, such as cytochromes P450, CYPOR, and nitric oxide synthase isozymes, because they harbor heme and/or flavin cofactors. ${ }^{1,11}$

Supporting Figure 1A demonstrates a spectrum of CYPOR with prominent absorption bands of the aromatic amino acids as well as the flavin adenine dinucleotide (FAD) and flavin mononucleotide (FMN). A choice of the donor-acceptor pair in a relatively absorption-free redinfrared range (Supporting Figure 1B) would effectively isolate FRET between the donor and acceptor from interference by intrinsic fluorophores. ${ }^{5}$ However, the major obstacle impeding fluorescencebased distance analysis in proteins is the difficulty of making pure donor-acceptor (DA) samples (not contaminated with a variable fraction of donor-only, DD, and acceptor-only, AA, species), which renders conventional FRET measurements based on fluorescence intensities largely qualitative. The time-domain experiments were shown to be capable of separating the DA signal from DD and AA 
contributions, thus allowing the donor-acceptor distance estimates in the mixtures. ${ }^{12}$ However, very short life times of the far-red fluorophores available for protein labeling ( $\sim 1 \mathrm{~ns})$ made time-resolved fluorescence measurements difficult. To circumvent this problem, we utilized ultrafast transient absorption pump-probe spectroscopy featuring a time resolution of 100 fs to accurately quantify FRET between the short-lived red fluorophores and to extract donoracceptor distance distributions. Ultrafast transient absorption pumpprobe spectroscopy has been widely used for studies of excitation and ET events in photosynthetic and other redox systems ${ }^{13}$ but has not been previously employed for quantitative distance analysis in protein structures.

The conformational states of the cytosolic domain of CYPOR are intensely debated with the closed state or a mixture of closed and open states detected for the oxidized CYPOR in solution, ${ }^{14-16}$ while Hedison et al. ${ }^{5}$ reported both fully oxidized and fully reduced CYPOR forms populating the open state. To quantitatively evaluate CYPOR conformational states through FRET detected via transient absorption, we utilized Alexa 647 and Alexa 750, the red donor and infrared acceptor, respectively. To maximize the sensitivity of FRET for the conformational state of the protein, we engineered the cysteine-less version of rat CYPOR with newly introduced cysteine residues in place of Q157 (FMN domain) and N271 (FAD domain), two surface residues separated by 43 and $63 \AA$ (the $C \beta-C \beta$ distance) in the closed and open conformations, respectively. The long wavelength range of the donoracceptor pair, the high quantum yield of the donor, and the high extinction coefficient of the acceptor resulted in a large Förster radius of $68.5 \AA$ (for details and references, see the Supporting Information, Calculation of Förster Radius). Therefore, we expected significant FRET in the CYPOR molecule in both conformations. Panels $A$ and $C$ of Supporting Figure 3 depict FRET in the oxidized cytosolic domain of CYPOR labeled with a donor-acceptor mix (DA sample) as an emission peak of the acceptor excited at the donor wavelength. To verify that the energy transfer between the donor and acceptor occurs only when they are present in the same CYPOR molecule, we created the "noFRET" mixture of the DD and AA (donor-only and acceptor-only) samples, which demonstrated no intermolecular FRET (panels B and C of Supporting Figure 3).

Biochemistry, Vol 55, No. 43 (November 1, 2016): pg. 5973-5976. DOI. This article is @ American Chemical Society and permission has been granted for this version to appear in e-Publications@Marquette. American Chemical Society does not grant permission for this article to be further copied/distributed or hosted elsewhere without the express permission from American Chemical Society. 
To quantify FRET between the donor and acceptor in the CYPOR molecule, we utilized femtosecond transient absorption spectroscopy (TA). The TA spectra (Figure 1) report the difference between the sample absorbance with and without excitation. The femtosecond laser excitation pulse (pump) at $600 \mathrm{~nm}$ promotes the donor molecules from their ground state to the excited state, thus depleting the population of the ground state, which is observed as increased transmission at 655 $\mathrm{nm}$ [ground state bleach (GSB)]. The donor molecules promoted to the excited state give rise to new positive absorption bands between 400 and $550 \mathrm{~nm}$. The isosbestic point at $570 \mathrm{~nm}$ indicates that the excitation and relaxation processes of the donor occur very close to the two-state transition ( $\left.\mathrm{D}+h \mathrm{v} \leftrightarrow \mathrm{D}^{*}\right)$. Both the GSB and the excited state absorption bands decay with similar kinetics (Figure 2).

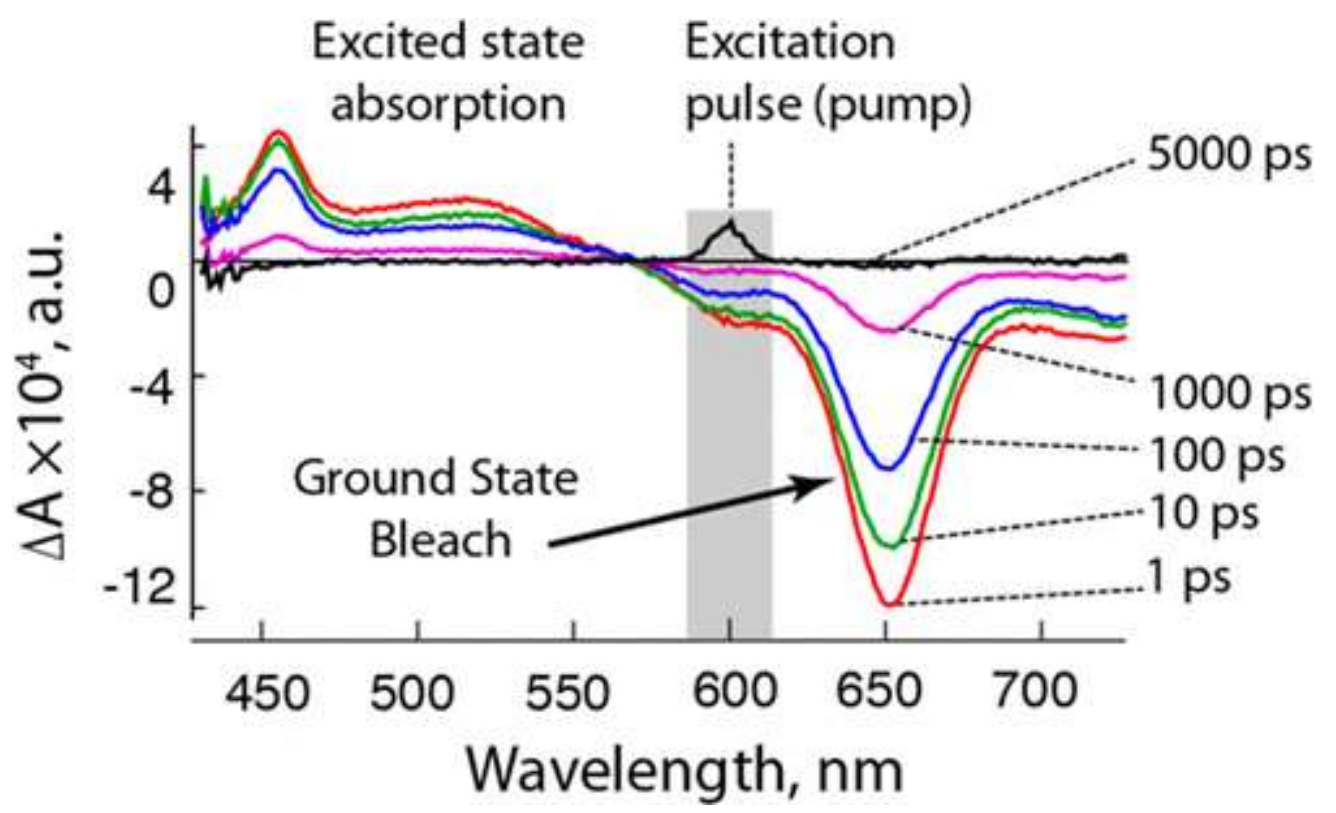

Figure 1. Representative femtosecond transient absorption spectra of the donoracceptor-labeled oxidized CYPOR at different time delays after $600 \mathrm{~nm}$ excitation. The gray bar indicates the spectral area distorted by the excitation pulse.

Biochemistry, Vol 55, No. 43 (November 1, 2016): pg. 5973-5976. DOI. This article is @ American Chemical Society and permission has been granted for this version to appear in e-Publications@Marquette. American Chemical Society does not grant permission for this article to be further copied/distributed or hosted elsewhere without the express permission from American Chemical Society. 


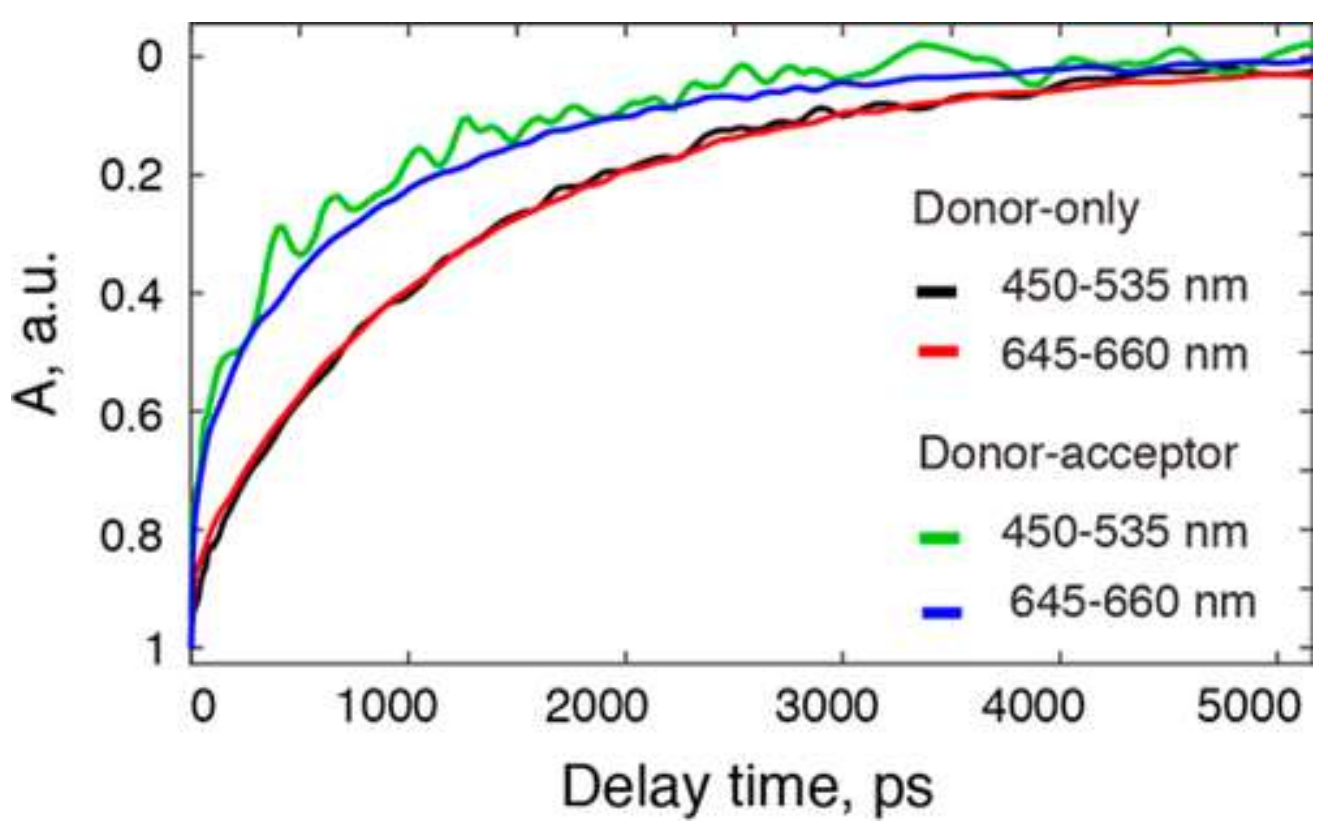

Figure 2. Kinetic traces of the ground state bleach recovery and excited state decay in the donor-only (DD) and donor-acceptor (DA)-labeled samples of oxidized CYPOR. The traces were obtained by averaging the range of wavelengths (indicated in the figure), calculating the running mean curve, and normalizing to unity at the maximal amplitude. The kinetic traces of the excited state were inverted to allow for direct comparison with the negative GSB decays.

In the presence of the acceptor fluorophore, the TA spectra demonstrate a faster decay of the GSB and the excited state features. Figure 2 shows the time course of the GSB recovery and the excited state decay (inverted for better comparison) of the DD and DA samples. The accelerated kinetics of the GSB recovery/excited state decay in the DA sample reflect the resonance energy transfer taking place between the donor and acceptor fluorophores when both are present in the oxidized CYPOR molecule. A similar pattern was also observed in the reduced CYPOR samples.

Because the $600 \mathrm{~nm}$ pump pulse may also weakly excite the acceptor, the possibility of the contribution of the acceptor spectral features, resulting from direct excitation, to the donor peak exists. Supporting Figure 4 shows that kinetics in the "no-FRET" sample (where donors and acceptors are attached to different CYPOR molecules) are nearly identical to those of the DD sample, implying that the acceptor makes a negligible contribution to the TA spectra at the donor wavelength. 
The major contributors to the GSB of the DA sample in Figure 2 are the (desired) donor-acceptor and (undesired) donor-only species. The kinetics of the DA and DD decays are expected to be very distinct because the Förster radius is comparable to or greater than the donoracceptor separation in both conformational states of the protein, resulting in a large FRET contribution to the DA decay. The flexible nature of the donor and acceptor fluorophores and possible dynamics of the protein structure require analysis of the FRET enhancement observed in our samples in terms of not just a fixed donor-acceptor distance but the distribution of possible distances. Analysis of timeresolved fluorescence in terms of Gaussian distributions of the donoracceptor separations in the sample was originally developed by Lakowicz ${ }^{12,17}$ and Albaugh and Steiner. ${ }^{18}$ We adapted their formalism for analysis of FRET detected via the TA measurements. Linearity of the absorption signal with concentration of the donor-labeled species allowed for straightforward separation of the DA from DD contributions to the data. Figure 2 demonstrates that the GSB (red and blue traces) had a signal-to-noise ratio much greater than that of the excited state decays (black and green); therefore, we utilized GSB traces for fitting with theoretical equations. The best-fit parameters are summarized in Supporting Table 1. For a detailed description of the method, see Materials and Methods in the Supporting Information and Supporting Figures 5-8.

Figure 3 displays molecular models of the crystallographically observed structural states of the cytosolic portion of CYPOR (panel A) and the averaged distance distributions resulting from fitting of duplicate DD and DA data sets for the oxidized and reduced CYPOR samples (panel B). The distance distributions describe the spatial separation between transition moments of the donor and acceptor, which are localized at fluorescent moieties of the Alexa 647 and 750 dyes. Considering the long, almost $10 \AA$ linkers connecting the fluorescent groups of the Alexa labels to the protein backbone, we expected distances relatively longer than the calculated separation of the cysteine side chains ${ }^{19}$ as well as significant widths of the distance distributions due to the flexible nature of the linkers. ${ }^{20}$ 


\section{A}
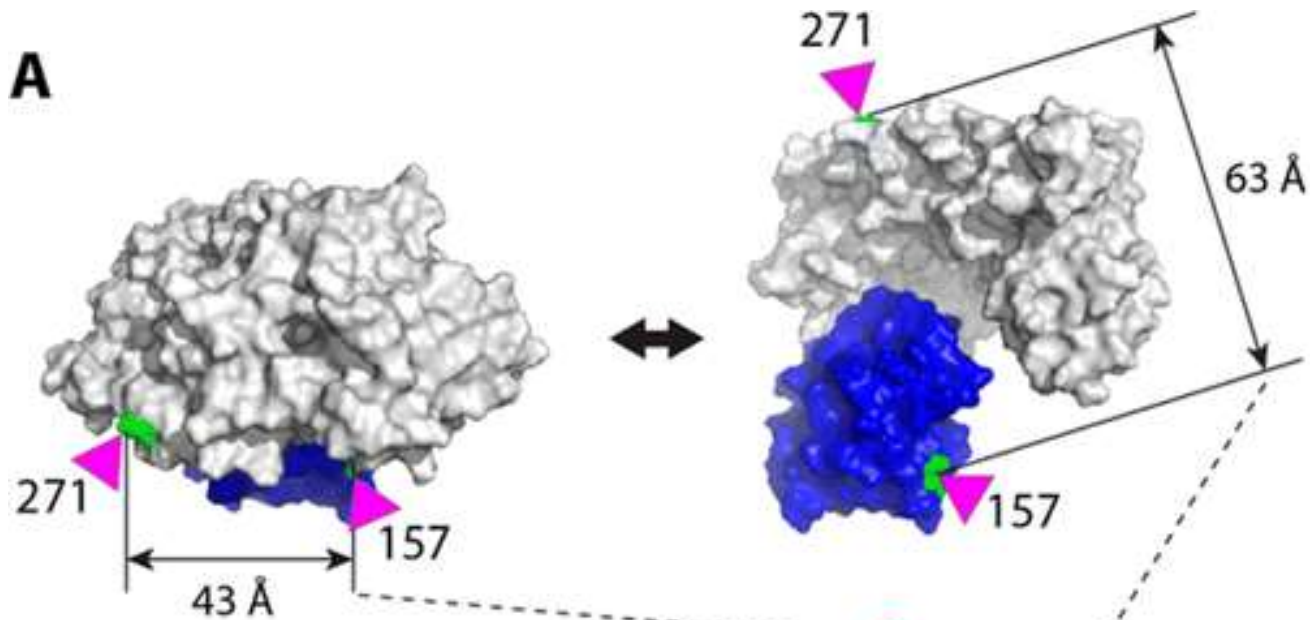

B

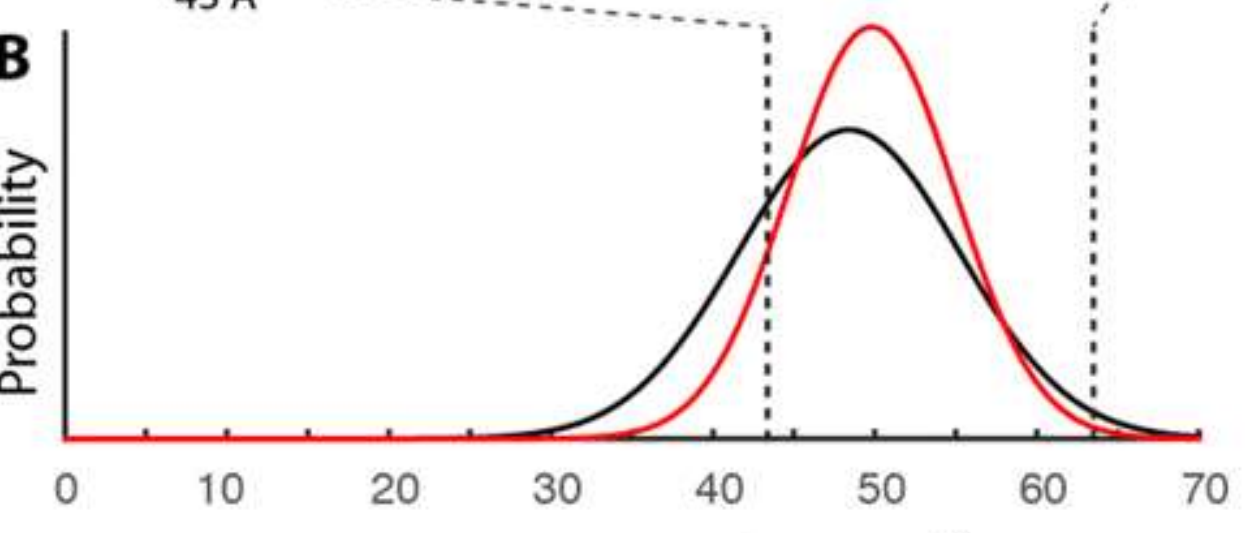

Donor-acceptor distance, $\AA$

Figure 3. Structural states of the cytosolic domain of CYPOR. (A) Molecular models of the closed form [left, Protein Data Bank (PDB) entry 1AMO] and the open form of CYPOR (right, PDB entry 3ES9, molecule A). The FAD-binding domain is colored gray and the FMN-binding domain blue. Pink triangles represent fluorescent labels (approximately to scale) at positions 157 and 271 (green surface). Distances between $\mathrm{C} \beta$ atoms of Q157 and N271 in two conformations are indicated. (B) Averaged distributions of distances between transition moments of the donor and acceptor fluorophores obtained from fitting the transient absorption data for oxidized (black) and reduced (red) states of CYPOR. For comparison, distances between C $\beta$ atoms of Q157 and N271 in the open and closed CYPOR models are indicated by vertical dashed lines.

The distribution mean of $48.4 \AA$ (distribution half-width of $16 \AA$ ) in oxidized CYPOR in Figure $3 \mathrm{~B}$ is consistent with the closed state of CYPOR, which is in full agreement with the literature reports. ${ }^{15,16}$ The distance distribution of reduced CYPOR in Figure 3B is slightly shifted toward the open form at $49.8 \AA$ (distribution half-width of $12 \AA$ ), yet the difference is small, implying that the reduced and oxidized CYPOR samples had statistically similar donor-acceptor separation in the absence of an electron acceptor (e.g., P450). It is also possible that 
$\operatorname{NADP}(\mathrm{H})$ binding favors the closed conformation of reduced CYPOR. In a kinetic FRET study, Hedison et al. ${ }^{5}$ found that the fully reduced state and the fully oxidized state have similar conformations based on FRET efficiency estimates. However, the authors did not measure distances and concluded that both states are fully open, contradicting other reports. ${ }^{15,16}$ The data presented here indicate that conformational states in both functional forms of CYPOR are similarly closed. Further studies are required to reveal how these conformational preferences of CYPOR are modulated by membrane binding and the presence of physiological ET partners.

In conclusion, we used ultrafast absorption spectroscopy of redinfrared donor-acceptor pairs to measure FRET in the flavoprotein CYPOR and assess its conformation in oxidized and reduced states. A simple labeling scheme with a mixture of reactive donor and acceptor fluorescent labels and superb time resolution of TA allowed for accurate recording of short-lived red-infrared fluorophore decays. We believe that this approach opens a new opportunity for quantitative distance analysis in CYPOR and other chromorphore-rich proteins in solutions as well as those bound to the phospholipid membranes.

\section{Author Contributions}

E.L.K., J.-J.P.K., J.H., C.X., E.A.K., and B.P. were involved in the conception and design of experiments. E.A.K., B.P., C.X., A.R.G., and E.L.K. performed the experiments. B.P. and E.L.K. analyzed the data. J.H., J.-J.P.K., and E.L.K. wrote the paper.

\section{Funding Information}

This work was supported in part by National Institutes of Health Grant GM097031 to J.-J.P.K. E.L.K. and J.H. acknowledge program development funds from Marquette University.

The authors declare no competing financial interest.

\section{References}

${ }^{1}$ Waskell, L. and Kim, J.-J. P. (2015) in Cytochrome P450. Structure, Mechanism, and Biochemistry (Ortiz de Montellano, P. R., Ed.) 4th ed., pp 33-68, Springer, Dordrecht, The Netherlands. 
NOT THE PUBLISHED VERSION; this is the author's final, peer-reviewed manuscript. The published version may be accessed by following the link in the citation at the bottom of the page.

${ }^{2}$ Wang, M., Roberts, D. L., Paschke, R., Shea, T. M., Masters, B. S., and Kim, J. J. (1997) Proc. Natl. Acad. Sci. U. S. A. 94, 8411-8416, DOI: 10.1073/pnas.94.16.8411

${ }^{3}$ Sugishima, M., Sato, H., Higashimoto, Y., Harada, J., Wada, K., Fukuyama, K., and Noguchi, M. (2014) Proc. Natl. Acad. Sci. U. S. A. 111, 25242529, DOI: $10.1073 /$ pnas.1322034111

${ }^{4}$ Pudney, C. R., Khara, B., Johannissen, L. O., and Scrutton, N. S. (2011)

PLoS Biol. 9, e1001222, DOI: 10.1371/journal.pbio.1001222

${ }^{5}$ Hedison, T. M., Hay, S., and Scrutton, N. S. (2015) FEBS J. 282, 43574375, DOI: $10.1111 /$ febs. 13501

${ }^{6}$ Forster, T. (1948) Ann. Phys. 437, 55- 75, DOI: 10.1002/andp. 19484370105

${ }^{7}$ Selvin, P. R. (2000) Nat. Struct. Biol. 7, 730- 734, DOI: 10.1038/78948

${ }^{8}$ Royer, C. A. and Scarlata, S. F. (2008) Methods Enzymol. 450, 79- 106, DOI: $10.1016 /$ S0076-6879(08)03405-8

${ }^{9}$ Hof, M., Hutterer, R., and Fidler, V. (2005) Fluorescence Spectroscopy in Biology. In Advanced Methods and their Applications to Membranes, Proteins, DNA, and Cells (Hof, M., Hutterer, R., and Fidler, V., Eds.) pp 3- 29, Springer, Berlin.

${ }^{10}$ Lakowicz, J. R. (2010) Principles of Fluorescence Spectroscopy, 3rd ed., Springer, Berlin.

${ }^{11}$ Poulos, T. L. and Johnson, E. F. (2015) Cytochrome P450. In Structure, Mechanism, and Biochemistry (Ortiz de Montellano, P. R., Ed.) pp 332, Springer, Dordrecht, The Netherlands.

${ }^{12}$ Lakowicz, J. R., Gryczynski, I., Wiczk, W., Kusba, J., and Johnson, M. L. (1991) Anal. Biochem. 195, 243- 254, DOI: 10.1016/00032697(91)90324-M

${ }^{13}$ Di Donato, M. and Groot, M. L. (2015) Biochim. Biophys. Acta, Bioenerg. 1847, 2- 11, DOI: 10.1016/j.bbabio.2014.06.006

${ }^{14}$ Ellis, J., Gutierrez, A., Barsukov, I. L., Huang, W. C., Grossmann, J. G., and Roberts, G. C. (2009) J. Biol. Chem. 284, 36628-36637, DOI: $10.1074 /$ jbc.M109.054304

${ }^{15}$ Vincent, B., Morellet, N., Fatemi, F., Aigrain, L., Truan, G., Guittet, E., and Lescop, E. (2012) J. Mol. Biol. 420, 296-309, DOI:

10.1016/j.jmb.2012.03.022

${ }^{16}$ Frances, O., Fatemi, F., Pompon, D., Guittet, E., Sizun, C., Perez, J., Lescop, E., and Truan, G. (2015) Biophys. J. 108, 1527-1536, DOI: 10.1016/j.bpj.2015.01.032

${ }^{17}$ Lakowicz, J. R., Gryczynski, I., Cheung, H. C., Wang, C. K., Johnson, M. L., and Joshi, N. (1988) Biochemistry 27, 9149-9160, DOI: 10.1021/bi00426a012

${ }^{18}$ Albaugh, S. and Steiner, R. F. (1989) J. Phys. Chem. 93, 8013-8016, DOI: 10.1021/j100361a011

Biochemistry, Vol 55, No. 43 (November 1, 2016): pg. 5973-5976. DOI. This article is @ American Chemical Society and permission has been granted for this version to appear in e-Publications@Marquette. American Chemical Society does not grant permission for this article to be further copied/distributed or hosted elsewhere without the express permission from American Chemical Society. 
NOT THE PUBLISHED VERSION; this is the author's final, peer-reviewed manuscript. The published version may be accessed by following the link in the citation at the bottom of the page.

${ }^{19}$ Majumdar, Z. K., Hickerson, R., Noller, H. F., and Clegg, R. M. (2005) J. Mol. Biol. 351, 1123- 1145, DOI: 10.1016/j.jmb.2005.06.027

${ }^{20}$ Klose, D., Klare, J. P., Grohmann, D., Kay, C. W. M., Werner, F., and Steinhoff, H.-J. r. (2012) PLoS One 7, e39492, DOI:

10.1371/journal.pone.0039492

\section{Supporting Information}

The Supporting Information is available free of charge on the ACS Publications website at DOI: 10.1021/acs.biochem.6b00623.

- Materials and Methods, calculations of the Förster radius, calculation of the fraction of signal from samples labeled with donor-acceptor mixtures, and supporting figures and references (PDF) 


\title{
Supporting Information
}

\section{Conformational states of cytochrome $\mathbf{P} 450$ oxidoreductase evaluated by FRET using ultrafast transient absorption spectroscopy}

\author{
Elizaveta A Kovrigina, Brian Pattengale, Chuanwu Xia, Azamat R Galiakhmetov, Jier Huang, \\ Jung-Ja P. Kim, Evgenii L Kovrigin
}

\section{Materials and Methods}

Protein construct To prepare the CYPOR construct with two exposed cysteine residues, we introduced sitedirected mutations in the "cysteine-less" construct of the cytosolic portion of rat CYPOR lacking 56 N-terminal residues of the transmembrane domain ${ }^{1}$. Mutation sites were chosen by criteria that the mutated residues must be significantly solvent-exposed, distant from the flavins, polar, uncharged, and exhibit a significant difference in inter-site distance upon CYPOR closed-open transition. The codons for Q157 and N271 were converted to cysteine codons using QuikChange II Site-Directed Mutagenesis Kit (Agilent) following the standard protocol. The expression plasmid included a linker encoding for six histidine residues in the $\mathrm{N}$-terminal linker to allow for $\mathrm{Ni}^{2+}$. affinity purification. In the following text, the resulting expression construct His6 Q157C Q517C CYPOR residues 57-678 is referred to as "CYPOR".

Protein preparation Expression and isolation CYPOR was performed as described earlier ${ }^{1,2}$. In short, the plasmid containing the expression construct was transformed into BL21star E. Coli bacteria, and the protein was expressed in Luria Broth medium upon induction with IPTG at room temperature. The culture was harvested, sonicated to lyse the cells, and centrifuged to remove insoluble proteins and the cell debris. The obtained supernatant was applied to the $\mathrm{Ni}^{2+}$-affinity resin, washed with $50 \mathrm{mM}$ imidazole buffer and eluted with $300 \mathrm{mM}$ imidazole

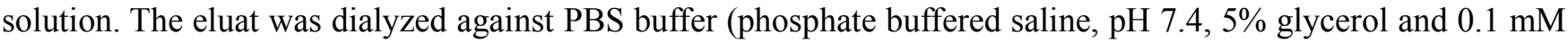
TCEP), loaded on the ADP-affinity column, and eluted with 2'-AMP. The final eluant was extensively dialyzed against PBS buffer resulting in the ligand-free CYPOR. Supporting Figure 2A demonstrates the final purity of the CYPOR preparation.

CYPOR samples for fluorescence and TA spectroscopy Alexa 647 and Alexa 750 maleimides (Life Technologies) were used to irreversibly attach Alexa fluorophores to the surface cysteines of the CYPOR construct according to the manufacturer's protocol. The total maleimide to cysteine ratio was kept at 10; ratio of Alexa 750 to Alexa 647 was $3 \mathrm{~mol} / \mathrm{mol}$ (for details on choice of the labeling ratio see Supp. Section 2). Labeling reactions contained $50 \mu \mathrm{M}$ total protein and were allowed to proceed overnight at room temperature in dark followed by separation of the reaction mix using the gel-filtration column Superose 6 Increase 10/300 (GE Healthcare Life Sciences) with Shimadzu HPLC system equipped with diode-array optical detector. Supporting Figure 2B shows a representative elution profile of the donor-acceptor labeled CYPOR sample. All protein samples exhibited a welldefined peak with the elution time of $38.5 \mathrm{~min}$ corresponding to monomeric CYPOR. The three preparations were made: donor-labeled, acceptor-labeled, and the sample labeled with the mixture of donor and acceptor (DA). The DA sample contains the donor-acceptor as well as donor-only and acceptor-only labeled molecules due to the statistical nature of labeling. The ratio of free maleimides in the reaction of $A: D=3: 1(\mathrm{~mol} / \mathrm{mol})$ was expected to produce a molar ratio of DD:DA:AA of 6:38:56 at 100\% labeling yield of available cysteines corresponding to the fraction of donor signal originating from DA species, $\mathrm{f}_{\mathrm{DA}}=0.75$ (see Section 2 below for calculation of $\mathrm{f}_{\mathrm{DA}}$ ). Practical labeling yield of cysteines in the purified samples were 0.82 and 0.94 as determined spectrophotometrically in two separate protein preparations, resulting in $\mathrm{f}_{\mathrm{DA}}$ of 0.59 and 0.67 , respectively (also see Correction for instability of the acceptor fluorophore). Typical CYPOR concentrations in the experiments were 3-6 $\mu \mathrm{M}$. Protein solutions were used directly from the gel-filtration tubes without additional concentrating. Solutions were spun at $15,000 \mathrm{~g}$ for 5 minutes prior to loading into the optical cells for measurements of the oxidized CYPOR samples. For preparation of the reduced CYPOR samples, NADPH was added to degassed samples of CYPOR under anaerobic conditions to final concentration of $0.5 \mathrm{mM}$. The reduced state of CYPOR was confirmed by disappearance of the flavin absorption around $460 \mathrm{~nm}$. The proteins were sustained in the fully reduced state during 4 hours 
of the TA experiments. Overnight storage led to complete re-oxidation of the samples by, presumably, the residual dissolved oxygen.

$U$ V-visible absorption spectroscopy Protein concentrations were established using both Pierce Coomassie Protein Assay Kit (Thermo Scientific) and absorbance at $460 \mathrm{~nm}$ with the extinction coefficient of $21600 \mathrm{au} \mathrm{M}^{-1} \mathrm{~cm}^{-}$ ${ }^{1}$. Concentrations of Alexa 647 and 750 fluorophores (Life Technologies) were determined using specific absorbance at their respective absorption maxima $\varepsilon(655 \mathrm{~nm})=265,000 \mathrm{au} \mathrm{M}^{-1} \mathrm{~cm}^{-1}$ and $\varepsilon(755 \mathrm{~nm})=290,000 \mathrm{au} \mathrm{M}^{-1}$ $\mathrm{cm}^{-1}$ respectively. For calculating the concentration of Alexa 647 in presence of Alexa 750, the absorbance of Alexa 750 at $655 \mathrm{~nm}$ was subtracted utilizing the experimentally determined ratio OD(Alexa 750, 750 $\mathrm{nm}) / \mathrm{OD}($ Alexa $750,655 \mathrm{~nm})=5.5$. All steady-state absorbance measurements were performed in 3 and $10 \mathrm{~mm}$ quartz cuvettes (Starna) using Varian Cary 50 Bio UV-Vis spectrophotometer.

Fluorescence spectroscopy Measurements of steady-state fluorescence were performed using Horiba (PTI) QM40 Spectrofluorometer. Standard one dimensional spectra were recorded with FelixGX software. Two dimensional spectra of the donor-acceptor labeled sample and the mix of donor-only and acceptor-only samples were recorded using custom FelixGX macro and visualized with Fluorescence2D sofware ${ }^{3}$.

Transient absorption spectroscopy (TA) The femtosecond TA measurements are based on a regeneratively amplified Ti-Sapphire laser system (Spectra Physics Solstice, $800 \mathrm{~nm}, 100 \mathrm{fs}, 3.5 \mathrm{~mJ} / \mathrm{pulse}$, and $1 \mathrm{kHz}$ repetition rate) and Helios spectrometer (Ultrafast Systems LLC). The $800 \mathrm{~nm}$ output from the regenerative amplifier was split to two parts with a beam splitter $(75 \% / 25 \%)$. The $75 \%$ part was used to generate tunable pump pulses $(235-$ $1150 \mathrm{~nm}$ ) through a TOPAS system. The energy of the pump beam was adjusted by various neutral-density filters. The pump light at $600 \mathrm{~nm}$ used in this work was set to $0.05 \mu \mathrm{J} /$ pulse. The other part of the beam was attenuated and directed to Helios spectrometer and used as probe beam. The white light continuum from $430 \mathrm{~nm}$ to $780 \mathrm{~nm}$ was generated by focusing $\sim 5 \mathrm{uJ}$ of the $800 \mathrm{~nm}$ pulse into a sapphire window. A synchronized $500 \mathrm{~Hz}$ chopper was used to chop the pump beam. The difference absorbance between the pumped and unpumped sample was recorded by the detector. The delay time between the pump and probe pulses was controlled by translational delay stage. The prepared samples $(0.5 \mathrm{ml})$ were held in a $2 \mathrm{~mm}$ quartz cuvette under continuous stirring during TA measurement.

Transient absorption data analysis The two-dimensional datasets of the differential absorbance as a function of the wavelength and time delay between pump and probe laser pulses were analyzed using Surface Xplorer (U1trafast Systems LLC) and the custom TA analysis module for the Integrative Data Analysis Platform software (available upon request from E. L. Kovrigin). To extract the time-dependent ground state bleach (GSB) decay, intensity values within $20 \mathrm{~nm}$ interval around the donor peak minimum $(655 \mathrm{~nm})$ were averaged for each time point, the early portion of datasets before $1 \mathrm{ps}$ was discarded, and maximum absolute intensity was normalized to unity.

Analysis of distance distributions from FRET The following analysis of exponential decays to obtain donoracceptor distance distributions taking into account incomplete acceptor labeling closely follows Lakowicz et al (1991) with little modifications ${ }^{4}$. The donor-only (DD) decays were fit with normalized triple-exponential functions:

$$
I_{D D}(t)=A\left(\left(1-a_{2}-a_{3}\right) e^{-t / t_{1}}+a_{2} e^{-t / t_{2}}+a_{3} e^{-t / t_{3}}\right) \quad \text { Sup. Eq. } 1.1
$$

To determine the FRET contribution to the donor GSB decay in the donor-acceptor (DA) samples, one takes into account that the GSB decay is a sum of decays of the donor-only, acceptor-only, and donor-acceptor CYPOR populations. The decays of the DD and AA species are measured directly using separate samples with only one kind of fluorophore added to the labeling reactions. Due to the low optical density of acceptor at the excitation pulse wavelength (600 nm; see Supporting Figure 1.B), the direct acceptor excitation was minimal, and therefore, the acceptor contribution to the GSB at the donor wavelength region $(655 \mathrm{~nm})$ was considered to be negligible. Contribution of the donor signal originating from the DD species may be separated from DA signal by considering the labeling yield of the sample:

$$
I=\left(1-f_{D A}\right) I_{D D}+f_{D A} I_{D A}
$$

where $f_{D A}$ is a fraction of the donor signal originating from the donor-acceptor labeled species. The $f_{D A}$ was calculated using estimated relative populations of the donor-acceptor species as well as the singly and doubly labeled donor-only species:

$$
f_{D A}=\frac{p_{D A}}{2 p_{D D}+p_{D A}+p_{D}}
$$

Sup. Eq. 1.3 
In contrast to fluorescence, where relative emission intensities are not proportional to concentrations of DA and DD species because quantum yield of donor in DA species is reduced due to FRET, the absorption is linear with the concentration of the species and their contributions to the observed signal may be estimated based on statistical reasoning (see Supp. Section 2).

Flexible attachment of the donor and acceptor fluorophores to the protein surface along with expected flexibility in the protein structure requires interpretation of FRET in terms of distance distributions ${ }^{5,6}$. In the first approximation, the donor-acceptor distance may be described by Gaussian law ${ }^{7}$ :

$$
\mathrm{P}_{R}=\frac{1}{\sigma \sqrt{2 \pi}} \exp \left(-\left[\frac{R-\bar{R}}{\sigma \sqrt{2}}\right]^{2}\right)
$$

where $R$ is the donor-acceptor distance, $\bar{R}$ - the distribution mean, $\sigma$ - the standard deviation. With the multiexponential decay of the donor, the donor signal in presence of the acceptor is ${ }^{5,6}$

$$
I_{D A}(t)=\int_{0}^{\infty} \sum_{i} a_{i} \exp \left[-\frac{t}{\tau_{i}}\left(1+\left(\frac{R_{o}}{R}\right)^{6}\right)\right] \mathrm{P}_{R} d R
$$

where $a_{i}$ and $\tau_{i}$ are amplitudes and life times of the donor decay in absence of the acceptor; $R_{o}$ is Förster radius.

The femtosecond excitation pulse in the ultrafast transient absorption instrument resulted in the width of the Instrument Response Function (IRF) of only $0.2 \mathrm{ps}$. Since the typical life times of the DD samples were much longer (ca. $8 \mathrm{ps}, 500 \mathrm{ps}$, and $1500 \mathrm{ps}$ ) and of the GSB decays were truncated before $1 \mathrm{ps}$, it was not necessary to calculate convolution of the IRF with the GSB decay allowing fitting the donor-only and donor-acceptor GSB decays directly with the Sup. Equations 1.1, 1.2, and 1.5.

Fraction of donor signal originating from DA species As we found from simulations with Sup. Equations 1.1, 1.2 , and 1.5 , the distance distribution parameters and $f_{D A}$ are strongly correlated and cannot be determined from fitting simultaneously. Therefore, it is important to have the $f_{D A}$ in the Sup. Eq. 1.2 obtained independently through measurements of the labeling yield and supplied as a constant parameter in the fitting session (see Supp. Section 2 for details of calculations). Sensitivity of the fitted distribution mean to accuracy of the experimental value of $f_{D A}$ was found to be modest: overestimation of the $f_{D A}$ by $10 \%$ in the fitting led to increase of the fitted distribution mean by $3-5 \%$.

Correction for instability of the acceptor fluorophore We observed that the Alexa 750 fluorophore gradually degraded in protein samples, particularly, under exposure to light in the fluorescence or TA spectrometer. The acceptor degradation was further accelerated in the presence of NADPH. Therefore, the experimental fraction of donor signal originating from DA species, $f_{D A}$, supplied to the fitting routine was determined from absorption spectra measured immediately prior to and immediately after the TA experiment. The obtained values were averaged and the resulting $f_{D A}$ was used in the fitting as a fixed parameter. Instability of the acceptor lead to decrease of $f_{D A}$ from approx. 0.59 and 0.67 immediately after preparation to 0.34 and 0.50 at the time of acquisition, to 0.25 and 0.34 after the measurements were completed for oxidized and reduced CYPOR samples, respectively. The values of $f_{D A}$ immediately prior to and after the TA experiments were averaged to result in respective $f_{D A}$ values of 0.30 and 0.42 , which were subsequently used as constant parameters in the fitting routine.

Molecular modeling The structures of the open and closed form of CYPOR were modeled in Pymol (https://www.pymol.org) from PDB entries 3ES9 (A molecule) and 1AMO, respectively.

\section{Calculation of fraction of the donor signal originating from donor-acceptor species, $f_{D A}$, when labeling with donor-acceptor mixtures}

When the protein construct with two solvent-exposed cysteine residues is labeled with a mixture of donor and acceptor maleimides (D and A) the resulting sample contains the following species: donor-acceptor labeled protein molecules, DA; protein molecules where both sites are occupied by the same label, DD and AA; incompletely labeled protein molecules with only one cysteine modified by the maleimide label, D-empty and A-empty. To 
calculate proportions of each kind of species in the resulting reaction product, we will make three basic assumptions: (1) both exposed cysteines are equally reactive (same rate constants for maleimide coupling); (2) donor and acceptor maleimides have equal reaction rate constants (fluorophore itself does not impact the coupling reaction rate); (3) labeling of two cysteines in one molecule is independent (one site with donor or acceptor does not bias probability of the other site to react with donor or acceptor). In this work, Assumption 1 is satisfied by the choice of similarly solvent-exposed sites on the surface of CYPOR (Q157 and N271). Assumption 2 is satisfied by the choice of Alexa 647 and Alexa 750 maleimides that are highly derivatized cyanine analogs and have high solubility. This is to be contrasted with sulfo-Cy5 and Cy7 maleimides that, while spectroscopically equivalent to the Alexa 647 and 750, have lower solubility resulting in significant under-labeling with the very hydrophobic Cy7. Assumption 3 is satisfied by selecting the two-cysteine protein construct with a very significant $(>20 \AA)$ distance between the sites to prevent direct physical interaction of the labels.

The following workflow enables determination of the fraction of the donor signal originating from the DA species in a mixture of DA, DD, and AA using experimental absorption spectra of the labeled protein samples (after purification by gel filtration to remove free labels):

1. Record the absorbance spectrum of the donor-acceptor sample and the separately prepared acceptorlabeled protein sample. Correct by subtraction of the buffer baseline.

2. Using acceptor-only labeled protein spectrum, determine the ratio of the acceptor Alexa 750 absorbance at the donor maximum wavelength of $655 \mathrm{~nm}$ to the absorbance at the acceptor maximum at $753 \mathrm{~nm}$ :

$$
\alpha_{\text {acceptor }}=A_{A A}(655) / A_{A A}(753) \text {. }
$$

3. Determine CYPOR concentration in the donor-acceptor sample, $C_{\text {protein }}$, using specific absorption of the fully oxidized flavins of 21,600 au M $\mathrm{M}^{-1} \mathrm{~cm}^{-1}$ at $460 \mathrm{~nm}$.

4. Determine the acceptor concentration in the donor-acceptor sample, $C_{\text {acceptor }}$, using the absorbance value at the peak maximum of Alexa 750, $A_{D A}(753)$, and specific absorption 290,000 au cm $\mathrm{M}^{-1}$ at 753 $\mathrm{nm}$. Donor Alexa 647 contribution to this wavelength is negligible.

5. Determine the donor concentration in the donor-acceptor sample, $C_{\text {donor }}$, using the acceptor absorbance at its peak maximum and the experimental absorbance at the donor wavelength:

$A_{\text {donor }}=A_{D A}(655)-\alpha_{\text {acceptor }} A_{D A}(753)$. Calculate the donor concentration using the donor specific absorption of $265,000 \mathrm{au} \mathrm{M}^{-1} \mathrm{~cm}^{-1}$ at $655 \mathrm{~nm}$.

6. Determine the total labeling efficiency, $y_{\text {labeled }}$, considering that each protein molecule has two exposed cysteines (for reporting the reaction progress; not used in further calculations):

$$
\begin{aligned}
& C_{C y s}=2 C_{\text {protein }} \\
& y_{\text {labeled }}=\frac{C_{\text {donor }}+C_{\text {acceptor }}}{C_{C y s}}
\end{aligned}
$$

7. Determine fraction of cysteines occupied by donors and acceptors:

$$
\begin{aligned}
& y_{D}=\frac{C_{\text {donor }}}{C_{C y s}} \\
& y_{A}=\frac{C_{\text {acceptor }}}{C_{C y s}}
\end{aligned}
$$

8. Fraction of unmodified cysteines is

$$
y_{\text {empty }}=1-y_{D}-y_{A}
$$

9. Considering that CYPOR construct has two cysteines, calculate probability (fraction) of each kind of labeled species using probabilities of labeling at each site.

The unreacted protein:

$$
p_{\text {empty-empty }}=\left(y_{\text {empty }}\right)^{2} \text {; }
$$

the single-donor labeled protein (counting both D-empty and empty-D species):

$p_{D}=2 y_{\text {empty }} y_{D}$;

both sites occupied by the donor: 
$p_{D D}=y_{D} y_{D}$;

the single-acceptor labeled protein:

$p_{A}=2 y_{\text {empty }} y_{A}$;

both sites occupied by the acceptor:

$p_{A A}=y_{A} y_{A}$;

donor-acceptor species (counting both D-A and A-D configurations):

$p_{D A}=2 y_{A} y_{D}$.

All these probabilities must sum to unity (use as a test for correct implementation of the computer code).

10. Calculate fraction of the donor absorption signal that originates from $\mathrm{DA}$ and $\mathrm{AD}$ species considering that DD species contribute the double amount of signal per unit of protein concentration (use Supporting Equation 1.3).

Note: if the labeling efficiency $y_{\text {labeled }}$ is lower than $100 \%$ the useful donor signal fraction will proportionally decrease.

The calculations suggest that increase of the A:D ratio in the labeling mix improves sensitivity to the DA signal by relatively reducing unwanted DD signal fraction. However, the absorption peak of acceptor Alexa 750 is quite broad; therefore, addition of A increases the acceptor contribution to absorbance at the donor wavelength thus placing a limit on the A:D ratio. We found that $A: D=3: 1$ molar ratio is a practical choice giving rise to $38 \%$ of DA (counting both D-A and A-D species) among all protein molecules while producing $6 \%$ of DD species. Considering double amount of absorbance originating from $\mathrm{DD}$ species, the labeling mix with $\mathrm{A}: \mathrm{D}=3: 1$ (after removing unreacted labels) results in the sample where $75 \%$ of donor signal is contributed by DA species with the remaining $25 \%$ coming from DD.

For comparison with the literature reports, we modeled the labeling reaction as conducted by Hedison et al. ${ }^{8}$ who utilized $\mathrm{A}: \mathrm{D}=1: 1$ and reported overall labeling efficiency of $y_{\text {labeled }}=0.19$. In this scenario, the authors had $65 \%$ of CYPOR remaining unlabeled, $16 \%$ of protein molecules in the form of D-empty, $16 \%$ as A-empty, $0.9 \%$ as $\mathrm{DD}, 0.9 \%$ as $\mathrm{AA}$, and $1.9 \%$ as $\mathrm{DA}$. The fraction of donor signal originating from DA species in this mixture is only $10 \%$, with most of the donor signal coming from D-empty. Considering that FRET leads to only a fractional change of the DA emission in the opening-closing transition, this explains why the fluorescence intensity changes did not exceed 3\% in the report by Hedison.

\section{Calculation of Förster Radius}

The Förster radius for the Alexa 647 - Alexa 750 donor-acceptor pair was calculated as prescribed by Lakowicz . Extinction coefficient of the acceptor $\left(290,000 \mathrm{au} \mathrm{cm}^{-1} \mathrm{M}^{-1}\right.$ at $\left.753 \mathrm{~nm}\right)$ and quantum yield of the donor (0.33) were reported by ThermoFisher (the Alexa dyes vendor). The emission spectrum of $1 \mu \mathrm{M}$ Alexa 647 in a PBS buffer was obtained with the excitation at $550 \mathrm{~nm}$ to record the full emission signal envelope. Calculation of the overlap integral of the emission spectrum of the donor and absorption spectrum of the acceptor (normalized to the value of the extinction coefficient at $753 \mathrm{~nm}$ ) was performed using $A E$ software package (kindly shared by Søren Preus; available from www.fluortools.com) and resulted in the value of $2.045 \times 10^{16} \mathrm{~nm}^{4} \mathrm{M}^{-1} \mathrm{~cm}^{-1}$. Calculation of the Förster Radius, $\mathrm{R}_{\mathrm{o}}$, assumed the refraction index of the buffer same as that of pure water $\mathrm{n}=1.4$. The isotropic orientation factor of $2 / 3$ was assumed as a good approximation because both labels are attached at the surface cysteines and separated from the protein surface by relatively long and flexible linkers. The resulting $R_{0}$ for the Alexa 647-750 donor-acceptor pair was $68.5 \AA$.

\section{Supporting References}

(1) Xia, C.; Hamdane, D.; Shen, A. L.; Choi, V.; Kasper, C. B.; Pearl, N. M.; Zhang, H.; Im, S. C.; Waskell, L.; Kim, J. J. J Biol Chem 2011, 286, 16246.

(2) Hamdane, D.; Xia, C.; Im, S. C.; Zhang, H.; Kim, J. J.; Waskell, L. J Biol Chem 2009, 284, 11374.

(3) Kovrigin, E. L. PLoS One 2014, 9, e101227. 
(4) Lakowicz, J. R.; Gryczynski, I.; Wiczk, W.; Kusba, J.; Johnson, M. L. Anal Biochem 1991, 195, 243.

(5) Albaugh, S.; Lan, J. Q.; Steiner, R. F. Biophysical Chemistry 1989, 33, 71.

(6) Albaugh, S.; Steiner, R. F. J Phys Chem-Us 1989, 93, 8013.

(7) Lakowicz, J. R.; Gryczynski, I.; Cheung, H. C.; Wang, C. K.; Johnson, M. L.; Joshi, N. Biochemistry 1988, 27, 9149 .

(8) Hedison, T. M.; Hay, S.; Scrutton, N. S. FEBS J 2015, 282, 4357.

(9) Lakowicz, J. R. Principles of Fluorescence Spectroscopy; 3rd ed.; Springer, 2010. 


\section{Supporting Figures}

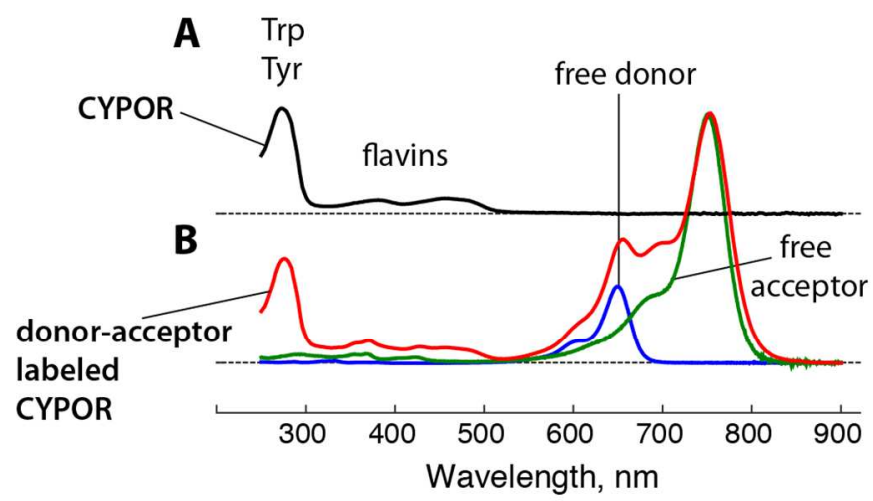

Supporting Figure 1. Absorption spectra of CYPOR (A) and the donor-acceptor pair used for distance measurements in this work (B). Donor, Alexa 647; Acceptor, Alexa 750. Labeling ratio (mol donor):( $m o l$ acceptor) $=1: 3$.

A

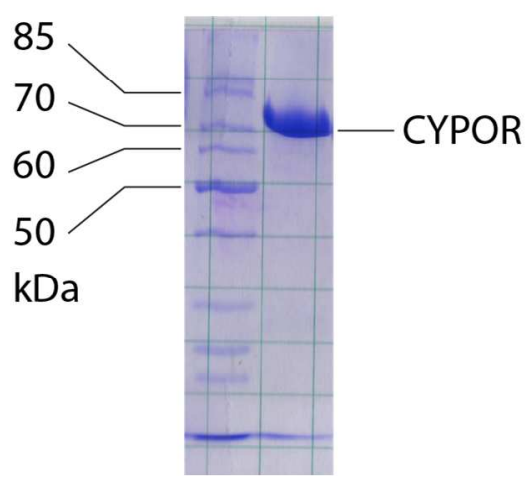

B

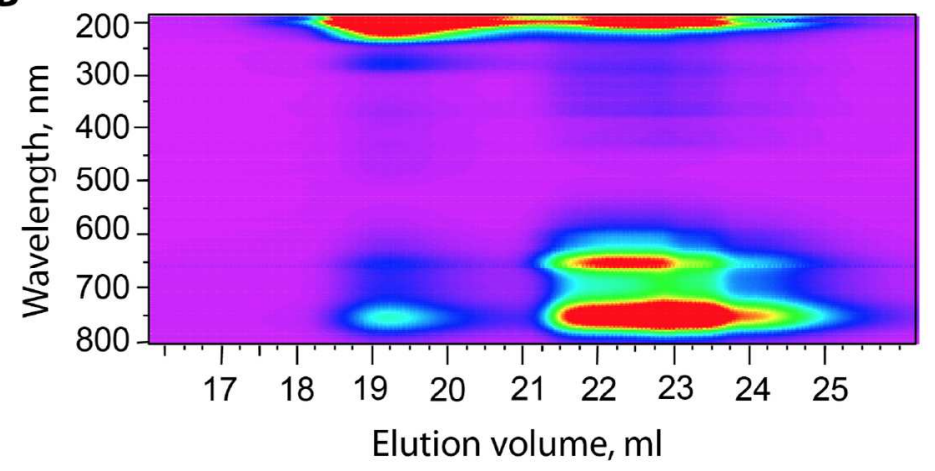

Supporting Figure 2. Panel A, SDS-PAGE of the final CYPOR sample prior to labeling. Panel B, Gel-filtration elution profile of the donor-acceptor labeled CYPOR sample using Superose 6 Increase 10/300 column in PBS, 5\% glycerol, $0.5 \mathrm{ml} / \mathrm{min}$. Void volume of the column was $10.5 \mathrm{ml}$, full volume $23.0 \mathrm{ml}$. Peak at $19.3 \mathrm{ml}$ corresponds to monomeric labeled CYPOR.
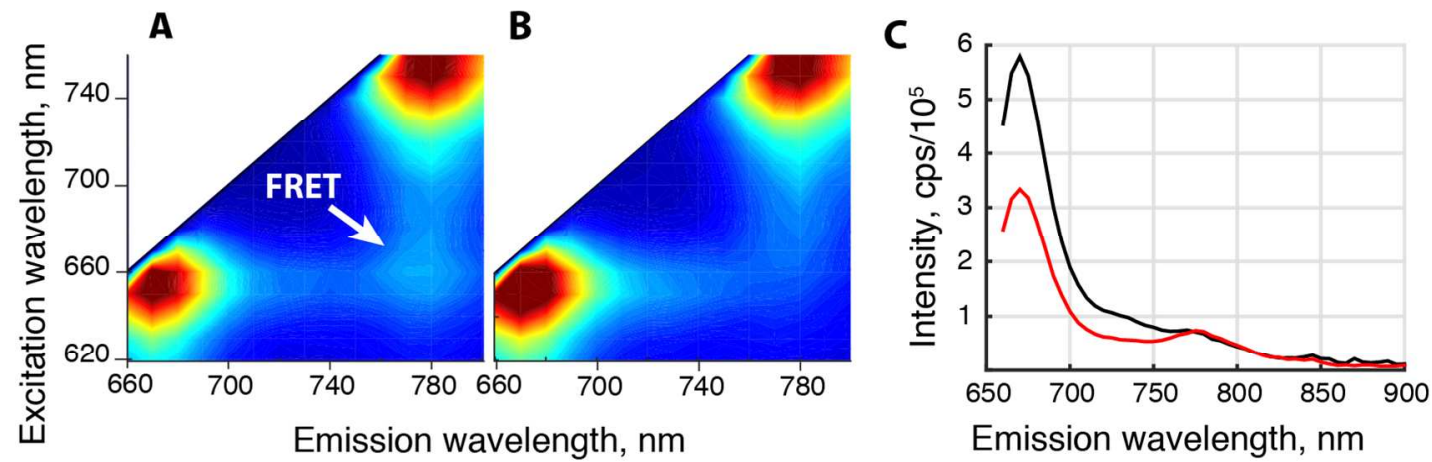

Supporting Figure 3. The representative two-dimensional fluorescence excitation-emission spectra of the oxidized CYPOR labeled with donor Alexa 647 and acceptor Alexa 750, (DA sample), A; and the mixture of donor-only and acceptor-only samples with the optical densities of the donor and acceptor matching those in the DA sample ("noFRET" sample), B. The one-dimensional fluorescence emission spectra of the DA (red) and "no-FRET" (black) samples excited at $650 \mathrm{~nm}, \mathbf{C}$. 


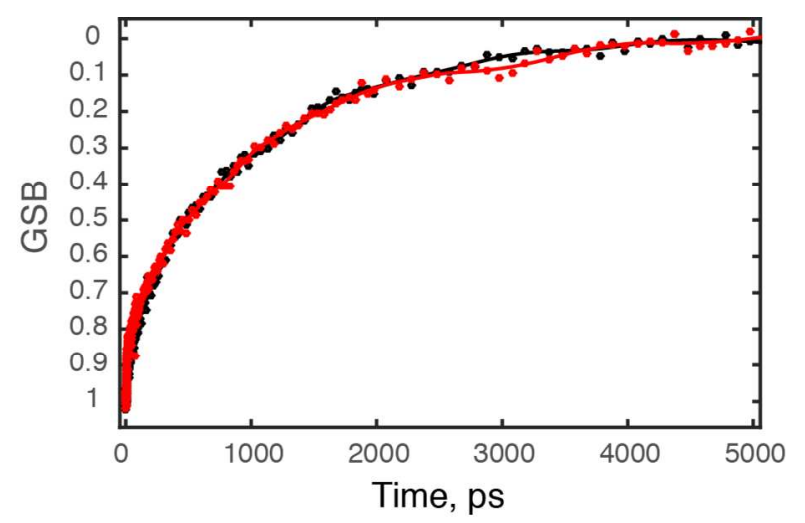

Supporting Figure 4. Comparison of GSB decays in the donor-only sample of the oxidized CYPOR, (black) and the "no-FRET" mixture made of donor-only and acceptor-only samples (red).
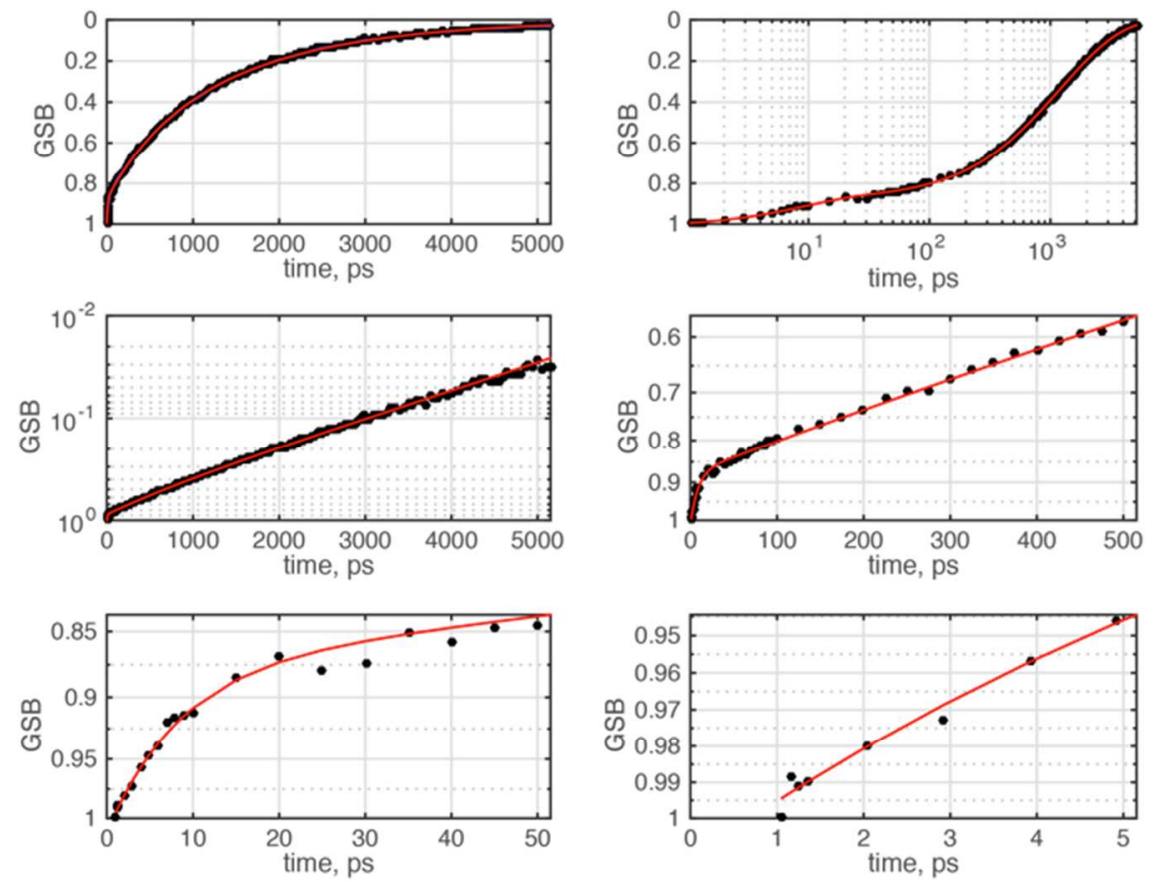

Supporting Figure 5. Fitting of the ground state bleach recovery of the donor-only labeled sample of oxidized CYPOR with a triple-exponential function (Sup. Eq. 1.1). Top panels demonstrate overall view of the signal in the linear and logarithmic time with the linear GSB axis. Middle and bottom panels show the logarithmic GSB signal in different time windows. Experimental data, black circles; best-fit model function, red line. Best fit parameters (95\% confidence intervals are given in square brackets): $A=1.01$ [ 1.001 .02 ], $t_{1}=8.5\left[7.59 .6\right.$ ] ps, $a_{2}=0.18\left[0.150 .22\right.$ ], $t_{2}$ $=510[450590] \mathrm{ps}, \mathrm{a}_{3}=0.68[0.640 .71], \mathrm{t}_{3}=1550[15201610]$. 

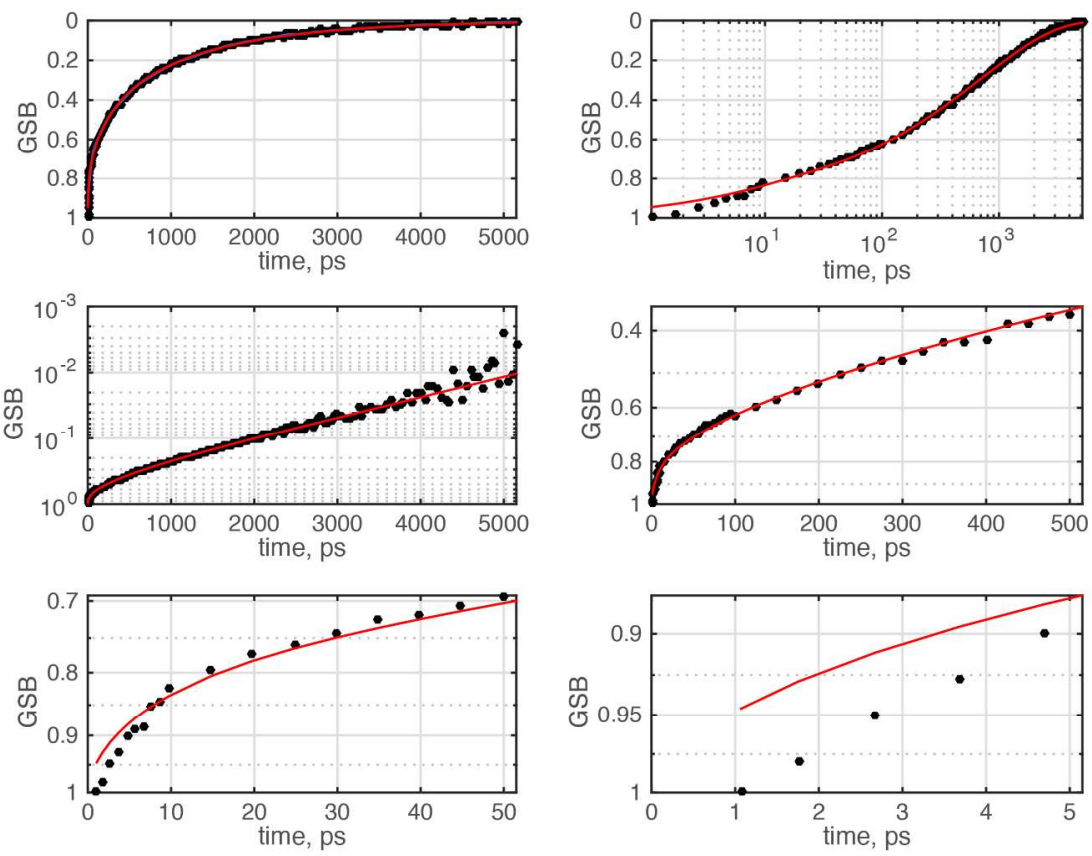

Supporting Figure 6. Fitting of the ground state bleach recovery of the donor-acceptor labeled sample of oxidized CYPOR with the donor decay law in the presence of acceptor assuming the Gaussian distribution of distances (Sup. Eq. 1.2-1.5). The amplitudes and life times of the donor decay in the absence of the acceptor were fixed to the best-fit values of the donor-only sample (Supporting Figure 5). The signal fraction originating from the DA species in the sample was estimated prior to experiment to be 0.50 while decreasing to 0.34 after acquisition. The two values were averaged to obtain $f_{D A}=0.42$ supplied to fitting routine as a fixed parameter. Experimental data, black circles; bestfit model function, red line. The distance distribution parameters shown in Figure 4.B were averaged from best-fit results of two pairs of DD and DA datasets.
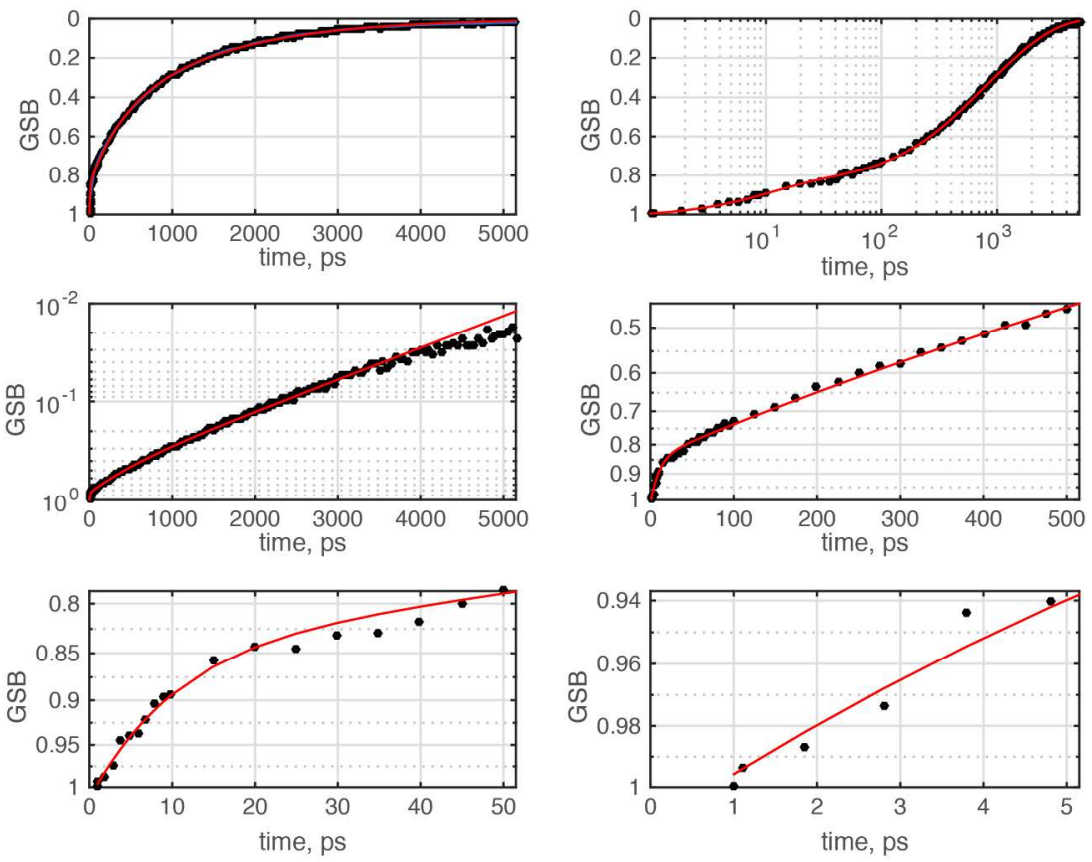

Supporting Figure 7. Fitting of the ground state bleach recovery of the donor-only labeled sample of reduced CYPOR with a triple-exponential function (Sup. Eq. 1.1). Best fit parameters (95\% confidence intervals are given in square brackets): $A=1.01[1.001 .02], t_{1}=9.5[8.510 .5]$ ps, $a_{2}=0.27[0.240 .30], t_{2}=510[450590] p^{2}, a_{3}=0.56[$ $0.530 .61], t_{3}=1340[13001370]$. 

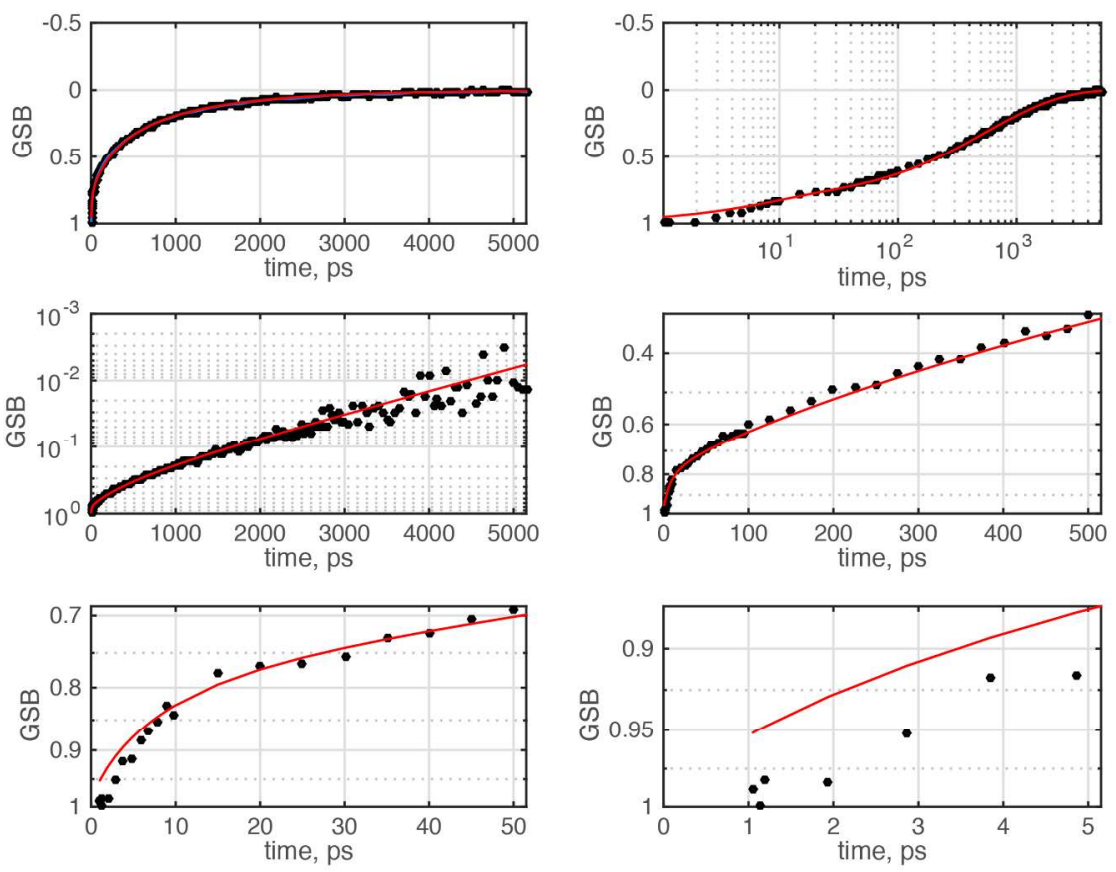

Supporting Figure 8. Fitting of the ground state bleach recovery of the donor-acceptor labeled sample of reduced CYPOR with the donor decay law in the presence of acceptor assuming the Gaussian distribution of distances (Sup. Eq. 1.2-1.5). The amplitudes and life times of the donor decay in the absence of the acceptor were fixed to the best-fit values of the donor-only sample (Supporting Figure 7). The signal fraction originating from the DA species in the sample was estimated prior to to experiment to be 0.34 while decreasing to 0.25 after acquisition. The two values were averaged to obtain $f_{D A}=0.30$ supplied to fitting routine as a fixed parameter. Experimental data, black circles; bestfit model function, red line. The distance distribution parameters shown in Figure 4.B were averaged from best-fit results of two pairs of DD and DA datasets.

Supporting Table 1. Best fit parameters of Gaussian distributions for the oxidized and reduced CYPOR averaged from fitting results of four pairs of donor-only and donor-acceptor TA decays.

\begin{tabular}{|l|c|c|c|c|}
\hline & Distribution mean, $\AA$ & $\begin{array}{c}\text { standard deviation } \\
(\mathrm{N})\end{array}$ & $\begin{array}{c}\text { Width at half height } \\
\text { of the distribution, } \AA\end{array}$ & $\begin{array}{c}\text { standard deviation } \\
(\mathrm{N})\end{array}$ \\
\hline $\begin{array}{l}\text { Oxidized CYPOR } \\
\text { (NADP-free) }\end{array}$ & 48.4 & $1.3(4)$ & 16 & $9(4)$ \\
\hline $\begin{array}{l}\text { Reduced CYPOR (in } \\
\text { the presence of } \\
\text { o.5mM NADPH })\end{array}$ & 49.8 & $2.2(4)$ & 12 & $3(4)$ \\
\hline
\end{tabular}

